\title{
Entrelacs
}

Cinéma et audiovisuel

\section{Le langage cinématographique et la codification du point de vue}

\section{Radhouan Maazon}

\section{(2) OpenEdition}

\section{Journals}

Édition électronique

URL : http://journals.openedition.org/entrelacs/149

DOI : 10.4000/entrelacs. 149

ISSN : 2261-5482

Éditeur

Éditions Téraèdre

\section{Édition imprimée}

Date de publication : 1 novembre 2005

Pagination : 138-150

ISBN : 978-2-912868-70-1

ISSN : 1266-7188

\section{Référence électronique}

Radhouan Maazon, «Le langage cinématographique et la codification du point de vue », Entrelacs [En ligne], 5 | 2005, mis en ligne le 01 août 2012, consulté le 19 avril 2019. URL : http://

journals.openedition.org/entrelacs/149; DOI : 10.4000/entrelacs.149

Ce document a été généré automatiquement le 19 avril 2019

Tous droits réservés 


\title{
Le langage cinématographique et la codification du point de vue
}

\author{
Radhouan Maazon
}

1 Précisons tout d'abord que l'expression: «langage cinématographique » désigne dans le discours sur le film aussi bien les paramètres plastiques de l'image que la question des raccords de plan à plan et les rapports de l'image et du son'1. Il s'agit donc, par analogie à la langue comme langage, de cette "grammaire» qui structure l'espace cinématographique sur les plans synchronique et diachronique. Toutefois, nous allons nous en tenir dans cette étude à l'approche diachronique de l'espace, voire à son déroulement spatio-temporel et ce, pour des raisons qui tiennent aux spécificités techniques et esthétiques du cinéma dont se soutient l'espace-temps qui, désormais se conçoit en tant que succession de plans, voire en tant que dialectique du champ et du hors-champ.

2 Mais de quel cinéma s'agit-il ? Il s'agit du cinéma dominant; celui qui domine la scène cinématographique par ses règles esthétiques, lesquelles règles commandent le passage de plan à plan par le recours au principe même du découpage invisible. Il s'agit en somme du cinéma légalisé, officialisé par l'institution, le commerce et la publicité.

3 Par conséquent, le présent texte se veut une étude en matière d'esthétiques cinématographiques faisant prévaloir le concept de «point de vue » en tant que concept structurant, lequel concept a servi d'outil d'analyse dans des développements antérieurs² ${ }^{2}$. Dans notre approche le concept de «point de vue » désigne a priori l'un des paramètres plastiques à savoir l'angle de prises de vues et, par voie d'extension, l'orientation prise par le cinéma en vue d'organiser et de structurer sa scène filmique par l'adoption de règles empiriques, donc esthétiques.

4 Nous allons donc observer dans les pages qui suivent, comment le cinéma du récit et de la narration s'emploie à codifier le "point de vue » de la caméra en s'organisant pour résorber cette cassure momentanée introduite au tournage en instaurant les lois et les règles qui participent à l'homogénéisation du récit. Ces règles se soutiennent de l'idée d'un « continuum » spatio-temporel et font du « point de vue » de la caméra un « point de 
vue » " coulé’ et «effacé » en allégeant les coupes, en les effaçant, en les rendant imperceptibles.

5 Il faut rappeler que dans des développements antérieurs ${ }^{3}$, nous avons pris comme point de départ, pour définir les paramètres plastiques de l'espace, le fait que ce dernier soit divisé : il y a le champ et le hors champ ; l'un étant le prolongement de l'autre, il en est le support imaginaire. Or, nous avons pu remarquer que le hors-champ fonctionne par la classification des plans selon la grosseur qui permet d'opérer une coupe à l'intérieur de l'espace profilmique, par le jeu des lignes et de perspectives, par l'incidence angulaire qui sélectionne une portion de cet espace, par le jeu de l'acteur qui jette des regards et qui se déplace, par la direction de la lumière principale et le lieu d'où elle émane.

Ainsi, l'écran cinématographique qui fonctionne dans les limites de la satisfaction et de la frustration, souligne et estompe au même moment. "L'image filmique n'est pas l'empreinte et le dépôt une fois pour toutes d'une réalité unique; affectée d'un manque, elle travaille (c'est l'histoire qui la fait travailler), creusée par ce qui ne s'y trouve pas $»^{4}$.

7 Certes, nous avons adopté une approche synchronique qui évacue l'élément durée et rythme et ce, à la manière de l'analyse qu'il est possible de mener à l'échelle du photogramme, ce qui nous a permis de nous arrêter sur les considérations plastiques de l'image. Ces dernières vont pouvoir se modifier dans la durée et avec le rythme du montage. "Changements de plans, mouvements de caméra et plasticité, montrent que la structuration de l'espace, si elle est fortement marquée par l'importance du cadre, relève aussi du passage d'un plan à un autre. En d'autres termes, il existe deux niveaux de structuration de l'espace: une structuration de l'espace cinématographique ou écranique (en fonction du cadre), et une structuration d'un espace qu'on pourrait appeler filmique. L'espace filmique est celui que constitue plan par plan, le film à travers son déroulement. Il ne s'agit pas alors de la composition à l'intérieur du plan, mais de l'espace à travers la succession des plans. $»^{5}$

8 C'est donc à l'intérieur de la succession des plans et par rapport à un cinéma tablant sur la recherche d'un continuum spatio-temporel sur l'axe diégétique, que le "point de vue » de la caméra s'est trouvé orienté selon un ensemble de «lois», de «codes» et de « conventions » afin de répondre à un mode de lecture qui suppose la " projection » sur l'écran d'un spectateur plus ou moins passif.

\section{Le « point de vue » de la caméra dans le même espace et le souci de continuité}

Ces «lois» ou «règles » vont porter sur le passage de plan à plan introduisant un changement de grosseur ou une variation angulaire, ce qui concerne donc les déplacements du "point de vue " de la caméra dans le même ensemble décor-acteur. Aussi, ces règles vont-elles, également, porter sur des variations de "point de vue " relatives à deux ensembles décor-acteur distincts. Il s'agit, en somme, de raccorder ces morceaux d'espace et de temps en prenant le soin de prévoir leurs raccords aux stades du découpage et du tournage. «Du point de vue formel, un film est une succession de tranches de temps et tranches d'espace. Le découpage est donc la résultante, la convergence d'un découpage dans l'espace (ou plutôt une suite de découpage dans le temps), prévu en partie au tournage et parachevé au montage. $»^{6}$

10 Restons au niveau du même ensemble décor-acteur et voyons les figures filmiques qui résultent du type de raccord porté sur le changement de grosseurs et sur les variations 
angulaires lorsque le sujet ou l'objet est fixe. Dans ce cas, il y a respectivement : « raccord dans l'axe » et « raccord avec variation d'axe».

\section{Le raccord dans l'axe et la règle du point de vue nettement différent}

11 Pour ce qui est du "raccord dans l'axe », le " point de vue » de la caméra est orienté suivant une règle qui amène à envisager la "suture » en fonction du paramètre de grosseur. Cette règle s'appelle: "la règle du point de vue nettement différent » Elle signifie que le changement de grosseurs, lorsqu'on passe d'un plan à un autre, suppose suffisamment d'amplitude, sous peine de créer une gêne pour l'œil du spectateur.

«Les raccords dans l'axe sont possibles si les plans successifs présentent entre eux des différences de taille. Sur un personnage, plan d'ensemble - plan moyen - gros plan, est une bonne succession, alors que plan américain large - plan américain serré - plan rapproché taille, crée après montage, une impression de piétinement insupportable, un hiatus visuel que seuls pourraient corriger de violents écarts angulaires. $»^{7}$

Notons que ces deux figures concernent le « raccord dans l'axe en avant » Dans le premier cas de figure, il y a respect de la "règle du point de vue nettement différent », dans le deuxième, il y a transgression de cette même règle. Et alors que le « raccord dans l'axe en avant» indique sur le plan spatial, le "rapprochement», le "raccord dans l'axe en arrière » indique "l'éloignement ». Ces sensations de rapprochement et d'éloignement nécessitent, selon le raccord dans l'axe, qu'il y ait continuité et non piétinement.

La continuité est ainsi, recherchée afin que l'œil ne soit pas choqué et gêné et ce, selon le souci du respect de la vision dite « réaliste $»^{8}$. D’ailleurs, le discours idéaliste sur le cinéma ne manque pas de souligner que tout raccord, même le plus invisible, le plus imperceptible, implique fatalement la fragmentation. Et il n'y a point de hasard à ce que les mouvements d'appareil aient fait irruption au moment où le montage constituait le fondement du langage cinématographique. Aussi, n'eut-il pas étonnant à ce que ce discours assimile, respectivement, les raccords dans l'axe en avant et en arrière et les travellings avant et arrière. "...Ces plans, pris dans le même axe, ne sont que des moments immobilisés d'une trajectoire mouvante, celle du travelling avant ou arrière. $»^{9}$

Pour commenter de telles affirmations, il y a lieu de préciser qu'il est d'une extrême naïveté d'assimiler un point de vue résultant d'une suite de cadres pris dans le même axe et celui que peut donner un travelling avant ou arrière, sachant bien sûr qu'une suite de plans fixes dans le même axe, signifie, sur le plan spatial et optique, un changement, voire un déplacement spatial quand il s'agit, par exemple, d'un raccord dans l'axe en avant, alors que le travelling avant produit, en nous, le sens de la découverte sous l'effet d'une modification des lignes de fuite qui s'écourtent à mesure que la caméra se déplace par chariotage.

Selon cette logique, le mouvement d'appareil, tout comme l'utilisation de la profondeur de champ, se substitue à une suite de plans sur une même scène même s'il y a respect de la « règle du point de vue nettement différent » Nous comprenons, aisément, à travers cette technique du tournage en un seul plan, ce souci de la continuité, comme si le cinéma est condamné, par essence, à ne fonctionner qu'aux termes de cette fonction unificatrice.

Aussi Philippe Durand a-t-il écrit : "...Alors que le mouvement d'appareil correspond en " un seul plan » à une attitude douée de réalisme, affectée de cette douceur de la logique qui s'échafaude peu à peu, le montage-choc des plans raccordés dans l'axe, a pour but de frapper, d'imposer par la 
violence, de traumatiser. Alors que le travelling adopte le cours d'une pensée discursive et permet l'identification, les raccords dans l'axe s'affirment dans le plus total arbitraire, comme des stimuli qui fondent sur les crânes et inhibent toute la liberté. $»^{10}$

18 Dans le même ordre d'idée, André Bazin en poussant au plus haut degré sa théorie du "réalisme», exprime une haine envers toute structuration spatiale à caractère fragmentaire: "Quand l'essentiel d'un événement est dépendant d'une présence simultanée de deux ou plusieurs facteurs de l'action, le montage est interdit. $»^{11}$

D'ailleurs, Bazin, n'hésite pas à faire le procès du montage : « ...il faut que l'imaginaire ait sur l'écran la densité spatiale du réel. Le montage ne peut y être utilisé que dans les limites précises, sous peine d'attenter à l'ontologie même de la fable cinématographique ».

Ainsi, nous pouvons saisir, chez Bazin, le fait que l'articulation montage-profondeur de champ se laisse comprendre en termes d'opposition et de conflit. Aussi, l'inscription historique de la profondeur de champ amène-t-elle à lire l'histoire du cinéma comme une histoire de conflit entre le « réalisme ontologique de l'image cinématographique », qui est le lieu de la "neutralité" de l'ambiguïté du réel et l'arbitraire des "significations imposées par le montage ».

\section{Le raccord avec variation d'axe et la règle des trente degrés}

21 Par ailleurs, si le « raccord dans l'axe " amène à penser la "suture » en fonction du paramètre de grosseurs, le « raccord avec variation d'axe " amène à penser la coupe en fonction de l'incidence angulaire. Or, ce type de raccord nécessite, pour qu'il soit bien réussi, qu'il y ait respect de la loi des trente degrés dont voici l'explication par Noël Burch :

22 "Il s'agit d'une règle qui s'est affirmée empiriquement pendant les années vingt, et qui veut que tout nouvel axe sur un même sujet differe de l'axe précédent par un angle d'au moins trente degrés. On s'était aperçu, en effet, que des changements d'angle de moins de trente degrés (à moins qu'il ne s'agisse d'un raccord dans l'axe), provoquent ce que l'on est convenu d'appeler un saut, une sorte de gêne due au manque d'ampleur et de netteté du changement intervenu : le nouveau plan n'a pas une autonomie suffisante surtout lorsque les grosseurs sont voisines... "

"Mais on pourrait aussi, affirmer que cette gêne provient de l'inutilité plastique d'un tel "raccord", et surtout d'une frustration de l'oeil qui demande des déplacements sensibles des contours d'un sujet, lorsque déplacement il y a, et que les tensions qui résultent de ces déplacements soient franches et nettes. $»^{12}$

Ce passage de N. Burch ne fait que pointer l'inscription esthétique et, par là même, idéologique de cette loi d'orientation. En effet, les années 20 ne sont pas sans rappeler un stade suffisamment élaboré du montage et de ses théories. Il s'agit, donc, d'une loi imposée par le cinéma narratif-représentatif qui en fait l'aboutissement d'un cinéma fondé sur le découpage. Ceci d'une part, d'autre part, cette règle n'est pas une règle scientifique mais empirique. Or, le fait qu'elle vienne s'ériger en une loi nécessaire par le raccord des espaces fragmentés et ce, en réponse à la « réalité de la vision » signifie que les défenseurs de cette règle partent du postulat selon lequel l'œil ne veut pas être frustré.

D'ailleurs, N. Burch qui relève dans une scène de La Ligne générale, une "suite de flashs ultra-courts de la canuelle de l'écrémeuse ", montre qu'Eisenstein opérait une transgression volontaire de la règle des trente degrés. 


\section{Une figure leurrante : « Le raccord dans le mouvement »} personnage et de tout mobile. Il appartient au monteur de repérer le moment, voire l'endroit opportun de la «coupe» pour que le geste soit lu dans la continuité à la projection.

32 Aussi, Ph. Durand retient-il, au montage, deux règles; l'une "d'équilibre »: le geste exécuté en deux plans ne se répartit pas moitié ; l'autre de " contraction » : le mouvement dans le plan 2 n'est pas repris à la place où il fut abandonné dans le plan 1 mais plus loin. A la collure entre les deux plans, doit s'opérer un phénomène de «saut ». Pratiquement, 
poursuit Ph. Durand, «il faut détecter sur la pellicule du plan 2, le premier photogramme dont la position correspond à celle du dernier photogramme sur la pellicule du plan 1; puis, ceci fait, couper une dizaine d'image en aval. Les deux tronçons du mouvement ne s'enchaînent souplement qu'au prix de cette amputation. Sinon, un effet de tamponnement, d'hésitation, se produit : les deux plans sont mal montés. $»^{15}$

\section{L'orientation du point de vue de la caméra et le principe de continuité}

33 Ainsi, il y a lieu de souligner que les « raccords dans le mouvement » ou les « raccords temporels » sont le prolongement affiné des « raccords dans l'axe » et des « raccords avec variation d'axe ». De même, le fait que ces raccords soient généralisés à partir de 1925, explique que les moyens de les réussir et de les obtenir se traduisaient dans les intentions qui animaient les esprits des cinéastes, des techniciens, des producteurs et distributeurs, en un mot, de tous les corps de métier qui se réclament de l'institution cinématographique dominante. Ce qui correspondaient, sur le plan du couple écriturelecture, à une volonté de redéfinir le processus de croyance chez le spectateur.

Or, à partir du moment où la mise au point technique de ces raccords fut imposée comme réponse à une demande esthético-idéologique, que le sentiment de continuité s'instaura comme une qualité qui manquait au film et que, par conséquent, on revendiqua la recherche d'une continuité à récréer à partir d'une discontinuité.

C'est en cela, que le discours théorique sur le cinéma vient doter les raccords temporels de significations intrinsèques. Aussi, le mouvement aller-retour de la main qui cherche la tasse pour la porter aux lèvres, donnerait-il cette impression de "statisme ", voire ces " effets de ciseaux », s'il était filmé en une seule prise de vue. "Par contre, vu l'un dans le plan 1, l'autre dans le plan 2, ils créent un dynamisme; les angles étant différents, il n'y a pas effet de redite, de piétinement, mais au contraire, structuration d'un mouvement. $»^{16}$

Il en est de même pour Jean Mitry, qui considère que la continuité constitue le fondement logique du drame : «...ce qui importe dans un film, hormis les qualités esthétiques (...) c'est le sentiment de continuité qui relie les plans et les séquences en maintenant l'unité et la cohésion des mouvements. La continuité logique ou dramatique dépend du scénario, mais il y a une continuité dynamique qui peut être envisagée ici $»^{17}$

37 A noter que la continuité dynamique, dont parle Mitry, n'a jamais fait l'objet d'un intérêt quelconque pour les cinéastes des premières années du cinéma, à plus forte raison, pour de nombreux cinéastes qui avaient, surtout, assigné à la caméra un tout autre " point de vue » et ce, à un moment où le cinéma instaura le découpage en continuité. Or, nous pouvons retenir respectivement, selon MITRY, le cas de Méliès et celui de Griffith.

En effet, Méliès, en ayant recours à un mode de narration, celui des "tableaux successifs ", ne se préoccupait guère de raccorder dans la continuité le mouvement contenu dans deux tableaux, dont le passage de l'un à l'autre, introduit une répétition que Mitry qualifie de « maladroite ».

39 "Lorsqu'on voulait suivre le même événement en passant d'un tableau à un autre » on devait reprendre " $L$ 'action en deçà du point de chute ou elle était arrivée. Ainsi dans 'le voyage à travers l'impossible » de Georges Méliès (1904) Le tableau $\mathrm{N}^{\circ} 4$ représente un paysage fortement accidente. On aperçoit L'automobile au professeur Maboulof qui dévale une pente à toute allure. A bout de course elle percute dans le mur d'une maison et pénètre à l'intérieur. 

succession des deux plans du messager par la «nécessité et 1 'intensité dramatique »: « Filmant les héros de près ou de loin, de face ou de profil, de dos ou de trois quart selon les points de vue successifs, il plaçait le spectateur au sein de l'action parmi les personnages du drame dans l'espace du drame. En saisissant Les choses selon des " points de vue » différents, le spectateur, en effet, les percevait tout comme s'il se déplaçait réellement autour d'elles. "L'impression de réalité " spatiale était de la sorte un fait acquis $»^{20}$

«Le tableau $N^{\circ} 5$ représente l'intérieur de la maison. Les occupants sont attablés et devisent joyeusement. Tout à coup, après avoir défoncé le mur qui s'écroule, une auto arrive en trombe et sème la panique parmi les convives qui s'enfuient épouvantés ${ }^{18}$.

IL y a dans ce passage de Mitry un souci de faire admettre l 'idée d'une histoire du cinéma qui est celle de la «continuité » et de la «vraisemblance » comme si de tout temps le cinéma était condamné à structurer ses espaces fragmentés selon 1 'impératif de "l'homogénéisation» de La scène filmique. Cette idée est bien visible dans Le remarquage par Mitry du premier geste inaugural : "Le premier sentiment de continuité fut donné dès 1902 par celui qui peut être considéré comme Le créateur du développement filmique l'Américain Edwin Stratton Porter $»^{19}$; aussi Mitry, ramène-t-il le "recours aux modes de narration du châtelet c'est à dire aux « tableaux successifs » à des contraintes techniques.

Or, si Méliès opère ainsi une mise bout a bout sans se soucier de couper dans le mouvement du tableau 4 pour le rattraper au tableau suivant, c'est qu'en toute évidence, pour lui en tant que prestidigitateur et illusionniste, le cinéma doit moins à l'artifice cinématographique qu'aux manipulations de décor, qui sont des tours tout simplement profilmiques.

De même, si nous voulons produire un nouvel indice du fait que l'inscription historique de la continuité n'est déterminée autrement que par des contraintes technicomatérielles, il importera de rappeler le choix fait par Griffith au moment où le cinéma opéra une restructuration de "l'impression de réalité » en instaurant, une fois pour toutes, la continuité comme un "gain de réalisme « qui manquait à la scène filmique. En effet, Griffith, conscient du "point de vue » qu'il voulait assigner a la caméra dans "Naissance d'une nation » présente une scène montrant un messager qui entre dans une salle de réunion lève le bras pour prendre la parole. Le premier plan montre le messager entrer dans le champ et lever le bras. Le second reprend sous une nouvelle incidence le mouvement intégral du personnage.

Dans cette scène il. n'y a pas de continuité dans le mouvement lors du passage du premier plan au deuxième pour la simple raison que la recherche de cette derrière n'était pas préoccupante chez Griffith qui voulait surtout se démarquer des schémas theâtraux et ce, loin de vouloir « choquer » L 'œil.

D'ailleurs étant soucieux de résorber la fragmentation, Mitry tente de justifier la Notons que «l'impression de réalité » intervient, chez Mitry, comme principe majeur de la représentation et de la lecture cinématographique. Encore une fois, se manifeste l 'idée de "camera-spectateur", celle qui opère des changements de grosseur et des écarts angulaires à l'intérieur du même ensemble décor-acteur. Selon cette conception, la restitution des éléments compositionnels est assurée de plans à plan ou de tableau à tableau même si le mouvement n'est pas raccordé lors du passage de L'un à L'autre. 


\section{Le " point de vue " de la caméra dans deux espaces distincts et l'instauration du mode de circulation de la parole.}

Cependant «si l'articulation des espaces hors-champ dans le même ensemble décor-acteur a nécessité » selon la règle de la continuité spatio-temporelle qu'il y ait suffisamment d'amplitude entre deux grosseurs consécutives", que les variations d'axe soient exécutées suivant la loi des 30 degrés et que les raccords dans le mouvement soient réalisés compte tenu des lois « d'équilibre » et de " contraction », il en est de même pour l'articulation du hors-champ dans deux ensembles décor-acteur distincts mais lisiblement contigus de plan a plan où le "point de vue de la caméra lors d 'une succession de champs alternés entre deux personnages, est codifiée par la règle des 180 degrés ».

\section{Le champ-contrechamp et la règle des 180 degrés}

Ainsi, cette figure, qui découle de la succession de deux champs alternés s'appelle: « champ-contrechamp ». Il s'agit d'une figure qui fonctionne massivement dans le cinéma de fiction et ce, au même titre que le raccord dans le mouvement. Aussi cette figure qui introduit un renversement des espaces dans un dialogue entre deux personnages demande-t-elle à être réalisée avec tellement de soin et de précaution sous peine de "dérouter" voire de "désorienter» le spectateur ». "Le champ-contre champ » introduisant une discontinuité de l'espace en cadrage opposés l'un a l'autre nécessite quelques précautions dans la manière de le réaliser. Il faut justement que dans ce renversement des champs "le spectateur ne perde pas pied et accepte sans malaise la succession des points de vue. Il faut que les espaces qui se font vis d vis" que la place des personnages " la direction de leurs regards de leurs déplacements obéissent à une logique conforme à celle que le spectateur reconstruit idéalement » $\mathrm{Ph}$. Durand ${ }^{21}$.

Nous comprenons aisément que le champ-contrechamp fonctionne selon le souci de la continuité qui n'est assurée qu'au prise du « respect « des « raccords « de regards de position et de déplacement qui tiennent à la prise en compte de la « règle des 180 degrés comme « loi » d'orientation du " point de vue » de la caméra. Or, le moyen de pallier la substitution brusque d'un ensemble décor-personnage par un autre ensemble, est d'opérer une obliquité des axes correspondant aux cadrages successifs. Cela revient à déplacer la caméra à l'intérieur d'un angle de 180 degrés qui peut être représenté en traçant un axe imaginaire reliant les deux ensembles décor-personnage.

Il faut préciser que cet axe délimite de part et d'autre deux demi-portions d'espace dans lesquelles la caméra peut se déplacer. Ainsi, afin d 'éviter la « déroute » et la " confusion « du spectateur quant à la direction du regard et à l'emplacement des deux interlocuteurs, cette «loi impose que l'opérateur se fixe par rapport à l'une des deux portions se tenant dans un angle de 180 degrés. A défaut du « respect « de cette consigne l'opérateur cadrerait le personnage $\mathrm{A}$ (qui est en regard par rapport au personnage B) à partir d'une portion d'espace et le personnage $\mathrm{B}$ à partir de la portion opposée ; nous avons donc un « faux raccord de regard » puisque les deux regards ne se croisent pas lors du passage du cadrage A au cadrage B à l'écran. 
51 Il faut également souligner que le " point de vue » de la caméra issu de cette règle des 180 degrés s'appuie sur des bases culturelle et anthropomorphique. Ainsi, l'orientation par cette règle des positions et des déplacements des personnages engage le transfert dans le domaine du film des repères théâtraux et des normes de la narration classique. Ceci d'une part, d'autre part, cette règle, en s'appuyant sur la fonction des regards des deux personnages, est donc programmée par cette base anthropomorphique.

Par ailleurs, il faut rappeler encore une fois que le recours au champ-contrechamp, voire au point de vue qui découle de la "règle des 180 degrés ", n'est qu'une conséquence de l'avènement $\mathrm{du}$ son et de la parole qui focalisent le principe même du découpage hollywoodien. Il s'agissait de réconcilier l'image et la parole ce qui ne fait que conditionner la lecture faite par le spectateur. Ici, le «point de vue » de la caméra est codifié de telle sorte qu'il scrute la source de la parole; il ne donne à voir que pour faire entendre. La parole et l'image fonctionnent en symbiose totale et le passage du champ au contrechamp correspond à un changement de répliques.

53 Aussi, faut-il souligner que le champ-contrechamp, qui est devenu avec le parlant la figure maîtresse d'un cinéma très "dialogué $~_{22}$, n'est pas tellement sollicité par les cinéastes de la nouvelle vague comme Godard, Resnais, Truffaut, Chabrol, Rohmer, et un peu plus tard Pialat, qui refusent de souscrire aux modes dominants de la narration. C'est ce dont témoignent les légers mouvements de caméra en aller et retour que Godard choisit d'utiliser pour filmer des scènes de dialogue dans A bout de souffle et Pierrot le fou ${ }^{23}$.

\section{Les règles de la continuité spatio-temporelle ou la recherche d'un " point de vue " « coulé » et " effacé »}

54 Notons par conséquent que les raccords de « regard « de " position « et d " "emplacement «qui se fondent sur la règle des 180 degrés au même titre que le raccord dans l'axe et le raccord avec variation d'axe qui nécessitent respectivement le « respect» de la « Loi du point de vue nettement différent « et la « loi des 30 degrés », caractérisent une pratique ayant pour fonction principale d'effacer le morcellement de recréer la continuité préalablement brisée donc de réunifier la discontinuité notamment au niveau de l'articulation des espaces hors-champ.

55 Aussi, le 'cinéma dominant" s'est-il employé durant l'évolution des techniques de découpage à instaurer ces règles tout en perfectionnant les méthodes permettant de les respecter afin de garantir la «suture» en effaçant les coupes et en rendant imperceptibles les changements de plans.

56 Comme le souligne N. Burch : "Avec l'avènement du son et l'accentuation du malentendu selon lequel le cinéma serait un moyen d 'expression "réaliste "» on est par-venu rapidement à une sorte de "degré zéro de l'écriture cinématographique»" tout au moins en ce qui concerne le changement de plan. Et les expériences de Russes qui avaient entrevu une toute autre conception du découpage » ont été vite considérées comme dépassées ou marginales. Le "faux-raccord » était a proscrire " au même titre que le raccord "pas clair " "puisqu'ils mettaient en évidence la nature ambiguë de l'espace cinématographique $»^{24}$. 


\section{Le « point de vue » d'une caméra mobile ou la recherche d'une continuité pure et immédiate.}

Cependant, avec la mobilité de la camera " même le "montage en continuité » est considéré comme une pratique contestable dans la mesure où ce montage introduit des effets de coupe et de rupture et ce » malgré le fait que les espaces discontinus sont rendus imperceptibles grâce à la « transparence des raccords ».

D’ailleurs, nous avons déjà précisé que les histoires et les théories du cinéma n'hésitent pas à assimiler : mise en raccord des espaces fragmentés et mobilité de la caméra. C'est ainsi que les mouvements panoramiques, en tant que mouvements de rotation autour des deux axes de la camera (axe vertical axe horizontal) se substituent, selon ces discours, à une suite fragmentée d'un même espace, et que les travellings, comme mouvements issus du déplacement latéral et frontal se substituent également aux morcellements issus des changements de grosseurs et des écarts angulaires.

De ce fait selon ces discours » comme selon un cinéma fondé sur la « vraisemblance « du récit et la continuité, le "point de vue» de la caméra donné par un mouvement panoramique ou par un travelling ou même par un mouvement combiné, renferme cette fonction franchement descriptive " surtout lorsqu'il s'agit de constater que ces théories associent la fonction narrative des mouvements d'appareil au tournage en une seule prise de vue. D'ailleurs, même le « mouvement d 'accompagnement «le suivi» que produit un panoramique horizontal, est utilisé à des fins descriptives. Cela se traduit, sur le plan narratif, par la « découverte progressive de la réalité », selon l'expression de Jean Mitry.

Par conséquent, afin de faire fonctionner le cinéma de façon prévalante à la « continuité » et à la « vraisemblance » du récit, il était prévisible que le «point de vue » de la caméra qui découle de la mobilité de cette dernière, soit codifié suivant des lois qui organisent la manière d'agencer les mouvements d'appareil, d'envisager leur assemblage dans une suite continue et de fixer leurs durées.

61 C'est ainsi, que dans l'agencement des mouvements d'appareil, il y a eu constamment une recherche du mouvement continu et « coulé » qui $n$ 'est rendu possible qu'avec le recours à des moyens techniques permettant une parfaite stabilité de l'appareil de prise de vue, voire une meilleure fluidité dans les mouvements de rotation de la camera autour de son axe, dans les déplacements par chariotage et surtout dans les mouvements de grue qui autorisent toute sorte de trajectoire. Aussi, le lancement de cette grue coïncide-t-il, sur le plan idéologique, avec cette "prise sur le réel » propre à l'homme dont le montage en discontinuité en est « l'acte perturbateur ».

Comme Le souligne, Ph. Durand, "en conférant la mouvance aux espaces cadrés, par conséquent en augmentant sensiblement la durée des plans, les mouvements d'appareil ont participé à cette évolution historique du langage qui se fit dans le sens de l'analyse à la synthèse, de l'effet montage à la fluidité ${ }^{25}$

63 Pour ce qui est de la succession de plusieurs mouvements de même nature, la consigne permettant de les raccorder veut que le sens d'orientation soit respecté. Or, ces mouvements ne se raccordent sans « heurts ni « contradictions visuelles » qu'à condition qu'ils soient de même sens. Ainsi, raccorder deux mouvements d'appareil de sens contraires (ex. un pano gauche-droite avec un pano droite-gauche) constitue la «faute » baptisée « ciseau ». 

travelling dans Week End de Godard ou le lent mouvement de grue ascendant dans : Il était Une Fois dans l'ouest de Sergio Leone, ou encore le lent panoramique circulaire dans Profession Reporter d'Antonioni, pour montrer jusqu'à quel point on peut faire jouer le couple frustration-satisfaction. Dans ces mouvements et trajectoires, il s'agit de retarder volontairement l'apparition dans le cadre de ce qui est encore hors-champ. Ainsi, dans Week End, le long travelling latéral d'une dizaine de minutes sur un bouchon de voitures retarde l'apparition de l'accident. Cette attente, écrit Marc Vernet, a ainsi pour conséquence de vider l'image d'une grande partie de sa substance, puisqu'elle n'est que transitoire : elle n'est jamais que le signe d'un passage ${ }^{26}$. clos et encombré, à l'immense désert de l'Ouest, se fait au moyen de cette lente trajectoire mouvante. Aussi, Antonioni a-t-il opéré un saut dans le temps donc un "flash forward " par le Long panoramique à 360 degrés dont le cadre de départ montre l'acteur Jack Nicholson cloué à son bureau et à gauche du cadre la fenêtre donnant sur un extérieur nuit. Alors que le personnage sort du champ à droite, la camera quitte le lieu par la gauche en balayant toute la pièce d'un mouvement circulaire jusqu'à revenir au point de départ où l'on voit en plongé à l'extérieur Jack Nicholson en état de vieillard qui s'éloigne à petits pas sous une nuit de neige.

Nous comprenons, aisément, que ni Godard, ni Léone, ni même Antonioni n'entendent, par ce parti pris à travers ces trajectoires, faire fonctionner le «point de vue » de la caméra dans un but descriptif. C'est dire qu'ils opèrent, par-là, une transgression de ce qui est défendu comme étant la « découverte progressive de la réalité » et la recherche de meilleures « conditions de visibilité ». Or, selon cette conception d'un cinéma narratif où la recherche de l'homogénéisation de l'espace constitue son fondement, des mouvements lents et trop longs ne font que «frustrer » le spectateur et compromettre «l'impression de réalité ».

\section{Conclusion}

Les développements précédents nous ont été nécessaires pour conduire l'analyse théorico-critique du cinéma vers la construction de la notion ou, si l'on veut, du concept de "point de vue» pour en faire le long du parcours un outil d'analyse historique, théorique et critique des formes et des figures cinématographiques et ce, en tâchant, à chaque instant, de cerner les déterminations esthético-techniques du cinéma.

Néanmoins, il faut tout de même préciser qu'une telle approche du cinéma n'a pas toujours constitué un principe de pertinence pour l'ensemble des historiens, théoriciens et critiques du cinéma qui admettent, volontiers, que le cinéma soit appréhendé du côté de son histoire, de ses genres, de son économie, de son public, mais jamais sinon rarement, du côté de ses formes qui, pourtant, le fabriquent de bout en bout.

A cet égard, la notion de " point de vue ", que nous mettons en avant dans cette étude, acquiert toute l'importance d'un outil d'analyse nous permettant de nous attarder sur les structures formelles qui sont en définitive, les structures plastiques et esthétiques de la scène filmique. Aussi, avons-nous souligner tout le long de cette étude, le fait que la notion de " point de vue » est fatalement liée à la notion de " hors-champ ». En d'autres termes notre démarche $d$ 'analyse nous a permis, d'abord, de conférer à l'espace hors-

Entrelacs, 5 | 2012 
champ une importance primordiale, ensuite, de préciser' que la manière d'envisager l'articulation des espaces hors-champ détermine un certain type de « point de vue » de la caméra.

70 Or, dans un cinéma, où la conception du hors-champ fait de ce dernier un prolongement du champ délimité par le cadre, il est évident que, ce qui fonde cette appréhension de l'espace en général, c'est l'idée d'un continuum spatio-temporel qui est à la base de ce qui est souligné être « l'équilibre » et « l'homogénéité » de l'espace dont se réclame le cinéma «dominant », qui fonctionne massivement du fait qu'il est légalisé et officialisé par le commerce et l'institution.

71 De même, ce cinéma, à tendance narrative, n'a pas manqué d 'assigner un « point de vue » à la caméra, lequel "point de vue» a été très fortement imprégné par la "vraisemblance» du récit et le "réalisme» dont se soutient tout "supplément » technique ayant marqué la mise au point du dispositif technico-matériel. En d'autres termes, tout supplément technique se traduit, idéologiquement, par un «gain de réalisme » et par une redéfinition des codes du « réalisme » en s'inscrivant comme une « vertu » qui manquait à la scène filmique.

72 C'est ainsi, que l'image grise et muette a cessé d'être le double reflet de la réalité et ce, au moment où en Amérique, l'irruption du son a précipité le lancement simultané de la panchromatique en noir et blanc et de la grue américaine.

73 Un peu plus tard, ce sera l'adjonction de la couleur et vers les années 50 la contre-attaque américaine se résoudra à la solution des procédés scopes et de l'écran large. D'ailleurs, ces suppléments techniques qui pouvaient être opérationnels bien avant leur mise au point définitive, ont eu lieu quand ceux qui s'en servent en ont besoin pour redéfinir l'impression de réalité et réactiver sans arrêt le «processus de dénégation » chez le spectateur.

74 Sur le plan des formes cinématographiques, la réaction américaine s'est déclenchée contre les pratiques du montage et de ses théories qui étaient à leur apogée vers les années 20. Afin de résorber la fragmentation et le morcellement de la scène filmique, Hollywood instaura simultanément les mouvements d'appareil et le découpage. Ce dernier admet l'antériorité du morcellement que le montage en continuité résorbera par la mise sur pied des lois et des règles qui, selon les impératifs de la continuité, organisent le passage de "plan à plan" selon les changements de grosseurs les incidences angulaires, la direction des regards, les positions et les déplacements des personnages. Aussi, le cinéma américain a-t-il établi des règles en vue de codifier l'usage des mouvements d'appareil suivant la reconduction des fonctions narrative et référentielle.

C'est de cette façon que le cinéma américain a pu organiser le morcellement de la scène filmique en codifiant le "point de vue" de la caméra suivant des règles rigides qui définissent son "essence » et sa "vertu». Il a, donc, choisi de vraisemblabiliser la division de la scène filmique en endossant le morcellement sur le compte de la diégèse. Or, par surcroit d'hégémonisme économique, culturel et idéologique, ce cinéma a su parler de la société américaine en développant des thèmes humanistes qu'il exporte dans le monde entier. Mieux encore, il s'agit tant pour Hollywood d'exporter, voire d 'universaliser un «mode de représentation «que d'envahir le marché mondial par ses films de fiction. A noter que ce phénomène d'homogénéisation du modèle hollywoodien acquiert toute son ampleur grâce à la diffusion de films par le biais des chaînes de télévision paraboliques. 

polarisent, essentiellement, autour de deux principales figures, à savoir le raccord dans le mouvement et le champ-contrechamp comme s'il existait une et une seule manière de filmer une conversation et ce, en alternant les champs respectifs aux deux interlocuteurs. Aussi, faut-il souligner que ce mode de représentation qui fait jouer en le codifiant le «point de vue» de la caméra, se fonde essentiellement sur une représentation du personnage héros. Or, greffer ce mode de structuration sur les cinémas nationaux, c'est aller à l'encontre de ce qui caractérise ces pays sur les plans de leur mode de distribution dans l'espace et leur manière de faire circuler la parole. D'ailleurs, nous voyons mal comment un « point de vue » articulant le champ-contrechamp rendrait-il compte du jeu dans des scènes de rue, de quartier, etc. à Tunis, au Caire ou a Khartoum... C'est dire que la fonction du héros telle qu'elle figure à travers cette figure filmique se rapporte a la culture et à la scène occidentale.

Ainsi, il y a lieu de remarquer que la notion de "point de vue » qui est déterminée par l'articulation du champ et du hors champ, dans un cinéma narratif représentatif, faisant prévaloir les règles de continuité spatio-temporelle, comme dans un cinéma se fondant sur le mécanisme de la coupe et de la faille, nous conduit à envisager le cinéma par rapport aux « modalités de représentation »

78 D'ailleurs, nous saisissons mal comment, face au discours diffus qui préconise un cinéma national qui traiterait des réalités authentiques, le cinéma du Tiers-Monde arrive-il à se démarquer du cinéma occidental s'il ne révise pas son mode de représentation. Car, en n'ayant pas à repenser la question du « point de vue » le message à produire serait trahi par un traitement qui adopte aveuglément une norme esthétique donnée.

79 Par conséquent, faire valoir la notion de point de vue c'est refuser de souscrire à l'idée, selon laquelle le cinéma est appelé a reproduire le monde, c'est quitter le degré zéro de l'écriture en vue de transformer le monde (la scène\} avec la production d'un sens nouveau; c'est surtout réexploiter les moyens qui tiennent franchement aux spécificités technico-esthétiques du cinéma

Il est temps que les « cinémas nationaux» (arabes, africains...) révisent leurs pratiques, tant sur le plan texte que sur le plan mise en scène, par une remise en cause des modalités de représentation qui ne sont guère les leurs. D'ailleurs, il y a lieu de constater que le débat portant sur les structures formelles de la scène filmique n'a jamais eu lieu dans les festivals arabes et africains du cinéma qui excellent dans la surenchère des débats stéréotypés tournant autour des conditions de production » et des circuits de distribution comme ce fut le cas des séminaires organisés lors des innombrables sessions des festivals, en l'occurrence les journées cinématographiques de Carthage ou le festival africain de wagadougou. Certes, les résistances ne manquent pas et il y a même une mise à l'abri volontaire et consciente des formes et des techniques, vis-a-vis des ideologies "dites subversives ». 


\section{NOTES}

1. http://fr.encyclopedia.yahoo.com/articles/ma/ma_3135_p0.html Décembre 2001

2. Le concept de point de vue nous a servi d'outil d'analyse dans deux études de recherche : 1- Pour une approche critique de la question du point de vue au cinéma, (en cours de publication) 2- La plastique du cinéma et la question du "point de vue" au cinéma, publiéé dans La Revue Tunisienne de Communication $\mathrm{n}^{\circ}$ 37-38 (2002).

3. Voir notre étude intitulée : "La plastique de l'image et la question du "point de vue" au cinéma ", publiée dans le Revue tunisienne de commmunication $n^{\circ}$ 37-38.

4. Pascal BONITZER : «Hors-champ » (un espace en défaut), Cahiers du cinéma n² 234-235 Jan - Fev 1972 p. 16.

5. Marc VERNET : "Structuration de l'espace » dans Lectures du film, Paris Ed. Albatros, Coll. Ça cinéma, Paris 1977.

6. Noel BURCH : “Comment s'articule l'espace-temps » dans : Praxis du cinéma, Paris Gallimard, Coll. Le chemin 1969, p. 12.

7. Philippe DURAND, L'Acteur et la caméra, Paris Ed. Techniques européennes, 1974, p. 129

8. Nous verrons qu'avec Méliès et Griffith, la continuité n'a jamais constitué un souci pour eux.

9. Philippe DURAND, L'Acteur et la caméra, op. cit. p. 129.

10. Dans : L'Acteur et la caméra, op. cit.. p. 130.

11. André BAZIN, Qu'est ce que le cinéma? (Montage interdit) Paris Ed. du Cerf $7^{\mathrm{e}}$ Art 1975 p. 59.

12. Dans : Praxis du cinéma, op. cit. p. 60.

13. La répartition du geste sur deux plans successifs signifie que le même geste est entamé dans le premier plan et achevé dans le deuxième.

14. Dans : L'Acteur et la caméra, op. cit., p. 157

15. Dans : L'Acteur et la caméra, op. cit., p. 157 - 158

16. Dans : L'Acteur et la caméra, op. cit.. p. 157

17. Jean MITRY : "Esthétique et psychologie du cinéma » Paris Ed. Universitaires Tome I, 1963, p. 157.

18. Jean MITRY, Esthétique et psychologie du cinéma, Tome II. Les formes (Ed. Universitaires -Paris 1965) p. 159. Page :93

19. Dans : Esthétique et psychologie du cinéma, Tome I op. cit.. p. 158.

20. Dans : Esthétique et psychologie Tome I. op. cit., p. 159

21. Dans : L'Acteur et la caméra, op. cit.. p. 8.

22. http :/www.france.diplomatie.fr/culture/france/biblio/folio/cinema/18.html, Décembre 2001

23. Godard dans A bout de souffle, choisit de passer de Jean Seberg à Jean-Paul Belmondo assis sur le lit dans la chambre par de légers mouvements de caméra, d'Anna Karina à Belmondo assis côte à côte dans une voiture par un court travelling latéral en aller-retour (au début de Pierrot le Fou, 1965). Même procédé pour une scène identique de Nous ne vieillirons pas ensemble (Pialat, 1972) entre Marlène Jobert et Jean Yanne. Le mouvement est alors plus ou moins motivé, voire pas du tout, par le dialogue. Il ne s'agit pas de filmer forcément celui qui parle et de revenir sur l'autre pour une réponse. Tous les décalages sont concevables comme le silence de celui ou celle qu'on ne voit pas pesant sur l'autre (Brigitte Sy et Anémone dans Baisers de secours de Garrel, 1989).

24. Dans : Praxis du cinéma ; op. cit. p. 22.

25. Dans : L'Acteur et la caméra, op. cit., p. 168.

26. Dans « Structuration de l'espace », (Lectures du film), op. cit., p. 92. 


\section{AUTEUR}

\section{RADHOUAN MAAZON}

Docteur en Études Cinématographiques. Département de Communication. Université du Roi

Saoud, Arabie Saoudite 